\title{
New Fractional Complex Transform for Conformable Fractional Partial Differential Equations
}

\author{
Y. ÇENESIZ AND A. KURT
}

\begin{abstract}
Conformable fractional complex transform is introduced in this paper for converting fractional partial differential equations to ordinary differential equations. Hence analytical methods in advanced calculus can be used to solve these equations. Conformable fractional complex transform is implemented to fractional partial differential equations such as space fractional advection diffusion equation and space fractional telegraph equation to obtain the exact solutions of these equations.
\end{abstract}

Mathematics Subject Classification 2010: 35R11, 34A08, 26A33.

Additional Key Words and Phrases: Conformable derivative; conformable fractional complex transform; fractional telegraph equation; fractional advection diffusion equation.

\section{INTRODUCTION}

Transform method which is applied in various fields is an important mathematical approach. With the help of this approach, partial differential equations which have difficult solution procedure can be converted into well-known differential equations which can be solved by known methods in the literature. Many transforms for solving these type problems are expressed in the literature such as the Laplace transform [1], the Fourier Transform [2], the Mellin transform [2]. The generalizations of differential equations with integer orders are called fractional differential equations. In recent years, interest to fractional differential equations has been increasing considerably because of its huge application area in various fields such as physics, engineering, dynamical systems, control systems $[2 ; 3 ; 4 ; 5]$. As a result of this interest, many powerful methods to solve fractional differential equations were presented by many authors. In addition to this, many researchers have been trying to form a new definition of fractional derivative such as Riemann-Liouville, Caputo, Gronwald-Letnikov [2; 3; $4 ; 5]$. Most of these definitions include integral form for fractional derivatives. Most popular ones are given following. 
(1) Riemann-Liouville Definition: If $n$ is a positive integer and $\alpha \in[n-1, n), \alpha$ derivative of function $f$ is given by

$$
D_{a}^{\alpha}(f)(t)=\frac{1}{\Gamma(n-\alpha)} \frac{d^{n}}{d t^{n}} \int_{a}^{t} \frac{f(x)}{(t-x)^{\alpha-n+1}} d x
$$

(2) Caputo Definition: If $n$ is a positive integer and $\alpha \in[n-1, n), \alpha$ derivative of function $f$ is given by

$$
D_{a}^{\alpha}(f)(t)=\frac{1}{\Gamma(n-\alpha)} \int_{a}^{t} \frac{f^{(n)}(x)}{(t-x)^{\alpha-n+1}} d x
$$

Nowadays a new definition of fractional derivative called "conformable fractional derivative" is presented by $\mathrm{R}$. Khalil and et al [6].

DEFINITON Let $f:[0, \infty) \rightarrow \mathbb{R}$ be a function. $\alpha^{\text {th }}$ order "conformable fractional derivative" of $f$ is defined by

$$
T_{\alpha}(f)(t)=\lim _{\varepsilon \rightarrow 0} \frac{f\left(t+\varepsilon t^{1-\alpha}\right)-f(t)}{\varepsilon}
$$

for all $t>0, \alpha \in(0,1)$. If $f$ is $\alpha$-differentiable in some $(0, a), a>0$ and $\lim _{t \rightarrow 0^{+}} f^{(\alpha)}(t)$ exists then define $f^{(\alpha)}(0)=\lim _{t \rightarrow 0^{+}} f^{(\alpha)}(t)$.

This new definition satisfies the properties which are given in the following theorem.[7]

THEOREM 1. Let $\alpha \in(0,1]$ and $f, g \alpha$-differentiable at point $t>0$. Then

(1) $T_{\alpha}(c f+d g)=c T_{\alpha}(f)+c T_{\alpha}(g)$ for all $a, b \in \mathbb{R}$.

(2) $T_{\alpha}\left(t^{p}\right)=p t^{p-\alpha}$ for all $p \in \mathbb{R}$.

(3) $T_{\alpha}(\lambda)=0$ for all constant functions $f(t)=\lambda$.

(4) $T_{\alpha}(f g)=f T_{\alpha}(g)+g T_{\alpha}(f)$.

(5) $T_{\alpha}\left(\frac{f}{g}\right)=\frac{g T_{\alpha}(f)-f T_{\alpha}(g)}{g^{2}}$.

(6) If , in addition to $f$ is differentiable, then $T_{\alpha}(f)(t)=t^{1-\alpha} \frac{d f}{d t}$.

\section{CONFORMABLE FRACTIONAL COMPLEX TRANSFORM}

Computing the numerical or exact solutions of conformable fractional differential equations is quite hard $[8 ; 9 ; 10]$. Therefore we apply a transform to get the solution of fractional differential equations easily. Thus the fractional differential equation can be converted into an ordinary differential equation. Recently, fractional 
complex transform for fractional differential equations with modified Riemann-Liouville derivative is introduced $[12 ; 13 ; 14 ; 15]$. In these article we introduce the new fractional complex transform for differential equations with conformable fractional derivative. In order to show the application of the transform, we handle the following fractional differential equation $[15 ; 16]$

$$
\frac{\partial^{\alpha} u}{\partial t^{\alpha}}=\kappa \frac{\partial^{\beta} u}{\partial x^{\beta}}, x \in \mathbb{R}, t \in \mathbb{R}^{+}
$$

with the initial condition

$$
u(x, 0)=2 x
$$

where $\kappa$ is a positive coefficient, $0<\alpha, \beta \leq 1$ and $\frac{\partial^{\alpha} u}{\partial t^{\alpha}}, \frac{\partial^{\beta} u}{\partial x^{\beta}}$ means the conformable fractional derivative of real valued function $u(x, t)$. For convenience we take $\kappa=1$. Conformable fractional complex transform is very easy to apply. First of all, let $\xi$ be an complex variable is defined as,

$$
\xi=a x^{\beta}+b t^{\alpha}
$$

where $a, b$ are constants to be found out further and $\beta, \alpha$ are fractional orders.

Substituting Eq. (3) in Eq. (1) by using chain rule [11], we get,

$$
(\beta a-\alpha b) u_{\xi}=0 .
$$

Firstly assume that $(\beta a-\alpha b) \neq 0$. Under this assumption, the solution is

$$
u(x, t)=c
$$

where $c$ is a constant. This trivial solution does not satisfy the initial condition (2). Then assume that $(a \beta-b \alpha)=0$. With the help of initial condition, we choose,

$$
u_{\xi}=\frac{2 \xi^{\frac{1}{\beta}-1}}{\beta a^{\frac{1}{\beta}}}
$$

and solving Eq. (6) we get,

$$
u(\xi)=\frac{2 \xi^{\frac{1}{\beta}}}{a^{\frac{1}{\beta}}} .
$$


Substituting Eq. (3) in Eq. (7) we have the exact solution which satisfies the initial conditions

$$
u(x, t)=\frac{2\left(a x^{\beta}+b t^{\alpha}\right)^{\frac{1}{\beta}}}{a^{\frac{1}{\beta}}}
$$

where $a$ and $b$ are parameters satisfying $(\beta a-\alpha b)=0$. By choosing $a=\frac{b \alpha}{\beta}$ the exact solution can be obtained as

$$
u(x, t)=\frac{2\left(\frac{b \alpha}{\beta} x^{\beta}+b t^{\alpha}\right)^{\frac{1}{\beta}}}{\frac{b \alpha}{\beta} \frac{1}{\beta}} .
$$

\section{SPACE FRACTIONAL ADVECTION-DIFFUSION EQUATION}

Now we consider space fractional homogenous advection diffusion equation of the form;

$$
\frac{\partial u}{\partial t}+\frac{\partial^{\alpha} u}{\partial x^{\alpha}}=\frac{\partial^{\alpha}}{\partial x^{\alpha}} \frac{\partial^{\alpha} u}{\partial x^{\alpha}}, t>0,0<x<a, 0<\alpha \leq 1
$$

where the fractional derivative is conformable fractional derivative [6].

By using conformable fractional complex transform

$$
\xi=m x^{\alpha}+n t
$$

we have the Eq. (10) as,

$$
m \alpha u_{\xi}+n u_{\xi}=m^{2} \alpha^{2} u_{\xi \xi}
$$

If we solve the Eq. (12) we get,

$$
u(\xi)=c_{1}+c_{2} \frac{m^{2} \alpha^{2}}{m \alpha+n} e^{\frac{m \alpha+n}{m^{2} \alpha^{2}} \xi} .
$$

Then substituting Eq. (12) in Eq. (13)

$$
u(x, t)=c_{1}+c_{2} \frac{m^{2} \alpha^{2}}{m \alpha+n} e^{\frac{m \alpha+n}{m^{2} \alpha^{2}}\left(m x^{\alpha}+n t\right)}
$$

where $c_{1}, c_{2}$ are integral constants.

As it is seen clearly, the solution can be easily obtained by the conformable complex transform. With respect to other analytical methods, conformable fractional complex transform has some advantages; 
1 - The ordinary differential equations obtained after applying the transform can be solved easily by the known methods in basic calculus.

2 - We can establish suitable boundary/initial conditions for governing equations with the help of this method. The numbers of boundary/initial conditions or the way they are obtained play an important role in fractional calculus. It may cause to non-existence of solution or ill-posed problem. For our problem we can consider the general initial condition as:

$$
u(x, 0)=a+b e^{c x}
$$

where $a, b, c$ are constants. Assume an initial condition in the form:

$$
u(x, 0)=e^{\left(x^{\alpha}\right)} .
$$

From Eq. (14), we have

$$
u(x, 0)=c_{1}+c_{2} \frac{m^{2} \alpha^{2}}{m \alpha+n} e^{\frac{m \alpha+n}{m^{2} \alpha^{2}}\left(m x^{\alpha}\right)} .
$$

Making comparison between the Eq. (16) and Eq. (17) implies that $c_{1}=0, c_{2}=\frac{1}{4}$, $m=4, n=-1$ and $\alpha=\frac{1}{2}$, hence we get the exact solution:

$$
u(x, t)=e^{\frac{1}{4}(4 \sqrt{x}-t)} .
$$

Unless the initial condition is stated in an exponential form, the least squares method can be used to get the constants in Eq. (14).[13]

\section{SPACE FRACTIONAL TELEGRAPH EQUATION}

Consider the following space-fractional telegraph equation:

$$
\frac{\partial^{2} u}{\partial t^{2}}+a \frac{\partial u}{\partial t}+b u=\frac{\partial^{\alpha}}{\partial x^{\alpha}} \frac{\partial^{\alpha} u}{\partial x^{\alpha}}, t>0,0<x<1,0<\alpha \leq 1
$$

where $a, b$ are constants, $u(x, t)$ unknown function and $\frac{\partial^{\alpha} u}{\partial x^{\alpha}}$ means $\alpha$ order conformable fractional derivative. Using the fractional complex transform

$$
\xi=m x^{\alpha}+n t
$$

then we have the Eq. (19) as

$$
n^{2} u \xi \xi+a n u \xi+b u=\alpha^{2} m^{2} u \xi \xi .
$$


Then solving Eq. (21) by known methods in classical calculus, we get;

$$
u(x, t)=c_{1} e^{\frac{\left(-a n-\sqrt{a^{2} n^{2}-4 b n^{2}+4 b m^{2} \alpha^{2}}\right)\left(m x^{\alpha}+n t\right)}{2\left(n^{2}-m^{2} \alpha^{2}\right)}}+c_{2} e^{\frac{\left(-a n+\sqrt{a^{2} n^{2}-4 b n^{2}+4 b m^{2} \alpha^{2}}\right)(m x \alpha+n t)}{2\left(n^{2}-m^{2} \alpha^{2}\right)}}
$$

\section{CONCLUSION}

In this paper we discuss about the usage of fractional complex transform for conformable fractional differential equations. It is easily seen that this transform is convenient for fractional partial differential equations and they are a good team with conformable fractional derivative definition. Conformable derivative definition is new and discussion of its role in fractional calculus is needed.

\section{REFERENCES}

[1] S. Kazem, International Journal of Nonlinear Science 16(1), 3-11 (2013).

[2] I. Podlubny, Fractional Differential Equations, Academic Press (1999).

[3] K. Oldham and J. Spanier, The Fractional Calculus, Theory and Applications of Differentiation and Integration of Arbitrary Order, Academic Press (1974).

[4] K.S. Miller, B. Ross, An Introduction to the Fractional Calculus and Fractional Differential Equations, A Wiley-Interscience Publication (1993).

[5] A.A. Kilbas, H.M. Srivastava and J.J. Trujillo, Theory and Applications of Fractional Differential Equations, Elsevier (2006).

[6] R. Khalil, M. Al Horani, A. Yousef, M. Sababheh, Journal of Computational and Applied Mathematics 264, 65-70 (2014).

[7] M. Abu Hammad, R. Khalil, International Journal of Pure and Applied Mathematics 94(2), 215-221 (2014).

[8] A.Kurt, Y. Çenesiz, O. Tasbozan, Open Physics 13 (1), 355-360 (2015).

[9] O. Tasbozan, Y. Çenesiz, A. Kurt, The European Physical Journal Plus, 131 (7), 244 (2016).

[10] O.S. Iyiola, O.O. Gbenga, Pramana 85 (4), 567-575 (2015)

[11] T. Abdeljawad, Journal of Computational and Applied Mathematics, 279,57-66 (2015).

[12] Z.B. Li, J.H. He, Nonlinear Sci. Lett. A,2(3), 121-126 (2010).

[13] Z.B. Li, W.H. Zhu, J.H. He, Thermal Science, 16(2), 335-338 (2012).

[14] J.H. He, Z.B. Li, Thermal Science, 16(2), 331-334 (2012).

[15] Z.B. Li, J.H. He, Mathematical and Computational Applications, 15(5), 970-973 (2010).

[16] G. Jumarie, J. Appl. Math. Computing 24(1-2), 31-48(2007). 


\section{Yücel Çenesiz}

Department of Mathematics, Faculty of Science

Selçuk University, Konya, Turkey

email: ycenesiz@selcuk.edu.tr

Ali Kurt

Department of Mathematics, Faculty of Science and Art

Mustafa Kemal University, Hatay, Turkey

email: alikurt@mku.edu.tr 